\title{
RADIAL FLOW THROUGH DEFORMABLE POROUS SHELLS
}

\author{
S. I. BARRY and G. K. ALDIS ${ }^{1}$
}

(Received 4 September 1991)

\begin{abstract}
The problem of radially directed fluid flow through a deformable porous shell is considered. General nonlinear diffusion equations are developed for spherical, cylindrical and planar geometries. Solutions for steady flow are found in terms of an exact integral and perturbation solutions are also developed. For unsteady flow, perturbation methods are used to find approximate small-time solutions and a solution valid for slow compression rates. These solutions are used to investigate the deformation of the porous material with comparisons made between the planar and the cylindrical geometries.
\end{abstract}

\section{Introduction}

When fluid flows through a porous material, the forces associated with the flow may deform the material. This deformation alters the properties of the porous material, which in turn affects the passage of fluid. There is therefore a complex coupling between the elasticity of the solid matrix and the fluid flow.

The first study of this was by Terzaghi [29] in 1925 and later by Biot [8] on fluid seepage from a consolidated soil. This led to the work of Biot [9, 10], used extensively in soil science. This theory has more recently been reformulated in terms of mixture theory to study the compressive behaviour of cartilage [24, 25, 26, 15] and arterial tissue [18, 20, 21]. The resultant nonlinear diffusion equation has been studied by Holmes [15, 13, 14] in application to stress relaxation and creep in articular cartilage. Comparisons of various models for the steady flow through a porous sponge [13] indicate that a simple finite deformation model with nonlinear permeability accurately

\footnotetext{
${ }^{1}$ Mathematics Department, University College, University of New South Wales, Australian Defence Force Academy, Canberra, A.C.T 2600, Australia.

(C) Copyright Australian Mathematical Society 1992, Serial-fee code 0334-2700/92
} 
models deformation behaviour. Barry and Aldis [3] have also examined the nonlinear behaviour of a deformable porous medium due to an unsteady applied pressure difference.

Although one-dimensional flow through a porous deformable slab has been considered in some depth, not as much is known about deformation in radial flow. Nevertheless, the radial flux of fluid through cylindrical shells is of importance as a model of arterial wall permeability [21] and in filtration technology [6]. The flow of proteins through the artery wall has been linked [28, 27] to the formation of atherosclerotic plaques (a form of arteriosclerosis). The deformation of the tissue may then be a factor in this process, especially if the nonlinear deformation is sufficient to impede the flux of proteins and hence cause them to accumulate. Filters can also be designed in the form of cylindrical shells [6] but no comparison has been made between planar and radial deformable filters.

The flow through an unconfined cylindrical shell of deformable porous material has been considered by Kenyon [21, 19] in estimating the deformation response after a step change in pressure. Jayaraman [17] extended this work to consider oscillatory flow, as did Jain and Jayaraman [16] who considered two materials joined together. In both of these works, boundary conditions were applied at the original boundary position rather than the final position; an infinitesimal assumption that will be investigated here. Klanchar and Tarbell [22] considered a simple nonlinear model for flow through a porous cylinder to find the steady state velocity and pressure relations.

In this paper we investigate the radial flow through cylindrical and spherical shells and compare these to planar flow. After deriving the governing equations and boundary conditions in a general form applicable to planar, cylindrical and spherical geometries, general solutions will be found for both steady and unsteady flow. These are used to compare the flow induced deformation in planar and cylindrical geometries and hence the filtration properties of these two geometries.

\section{Mixture theory for a two phase medium}

The theory of mixtures is based on the idea that the individual components of the mixture (here a solid matrix and a fluid) can be "modeled as superimposing continua so that each point in the mixture is occupied simultaneously by a material point of each constituent" [7]. The mixture theory outlined here is based on Bowen [11]. A detailed analysis of mixture theory is given in [2].

It is assumed that the fluid is a Newtonian viscous fluid and that the 
elastic solid matrix is homogeneous, isotropic and that there exist no external body forces or osmotic forces. Shear stresses are not considered since the flow is purely radial. The individual components of the mixture will be assumed to be intrinsically incompressible. Thus bulk compression arises from a reduction in the fluid volume fraction rather than compression of the individual constituents.

The volumes of the two constituents are denoted $V^{\beta}$ and where $\beta=s$, $f$ denotes either the solid or the fluid phase. The apparent densities of the constituents are given as

$$
\rho^{\beta}=\lim _{d V \rightarrow 0} \frac{d m^{\beta}}{d V},
$$

where $d m^{\beta}$ is the mass of the $\beta$ phase in the small volume $d V$. The true (or intrinsic) density of each phase is

$$
\rho_{T}^{\beta}=\lim _{d V^{\beta} \rightarrow 0} \frac{d m^{\beta}}{d V^{\beta}},
$$

where $d V^{\beta}$ is the small volume occupied by the $\beta$ phase. The relative volume fractions of each phase are

$$
\phi^{\beta}=\lim _{d V \rightarrow 0} \frac{d V^{\beta}}{d V},
$$

from which it is easy to see that

$$
\begin{aligned}
\rho^{\beta} & =\phi^{\beta} \rho_{T}^{\beta}, \\
d V & =d V^{s}+d V^{f}, \\
1 & =\phi^{s}+\phi^{f}, \\
\rho & =\rho^{s}+\rho^{f},
\end{aligned}
$$

The continuity equation for each phase can then be derived [26] as

$$
\frac{\partial \rho^{\beta}}{\partial t}+\nabla \cdot\left(\rho^{\beta} \mathbf{v}^{\beta}\right)=0
$$

where $v^{\beta}$ represents the velocity of the $\beta$ phase.

Since the the constituents are intrinsically incompressible, $\rho_{T}^{\beta}$ is constant and use of (4) in (8) yields

$$
\frac{\partial \phi^{\beta}}{\partial t}+\nabla \cdot\left(\phi^{\beta} \mathbf{v}^{\beta}\right)=0
$$

Adding the solid and fluid forms of this equation and using (6) leads to

$$
\nabla \cdot\left(\phi^{f} v^{f}+\phi^{s} v^{s}\right)=0
$$


The momentum equation for each phase can be written as

$$
\rho^{\beta}\left(\frac{\partial \mathbf{v}^{\beta}}{\partial t}+\left(\mathbf{v}^{\beta} \cdot \nabla\right) \mathbf{v}^{\beta}\right)=\nabla \cdot \mathbf{T}^{\beta}+\rho^{\beta} \mathbf{b}^{\beta}+\pi^{\beta},
$$

where $T^{\beta}$ is the stress tensor for the $\beta$ phase, $\mathbf{b}^{\beta}$ is the resultant external body force (neglected here) and $\pi^{\beta}$ is a drag force between the constituents representing internal forces due to frictional interaction between the two phases.

For small velocities and deformation rates, the inertial terms can be assumed negligible. Barry and Aldis [5] have considered the effect of the inertial terms in the derivation of the governing equations, and have shown that they may be assumed negligible if $t_{0}>k_{0} \rho_{T}^{s}$ where $t_{0}$ is the typical time scale and $k_{0}$ is a typical permeability of the medium to fluid flow.

With these assumptions, (11) becomes

$$
\nabla \cdot \mathrm{T}^{\beta}=-\boldsymbol{\pi}^{\beta},
$$

where Newton's third law implies $\pi^{s}=-\pi^{f}$; the force on the solid by the fluid is opposite to the force on the fluid by the solid.

The stress tensors can be modeled as

$$
\begin{aligned}
\mathbf{T}^{\beta} & =-\phi^{\beta} p \mathbf{I}+\sigma^{\beta}, \\
-\pi^{s}=\pi^{f} & =K\left(\mathbf{v}^{s}-\mathbf{v}^{f}\right)-p \nabla \phi^{s},
\end{aligned}
$$

where $\sigma^{s}$ represents a solid stress, the "contact stress" [18], a function of the strain, $K$ is the drag coefficient of relative motion, $p$ is the fluid pressure and $I$ the identity tensor. These stress equations split the stress tensors into contributions due to hydrostatic pressure and those due to viscous stress or solid matrix stress. The interaction term represents the linear drag between the constituents that is drawn from Darcy's law.

For one dimensional and radial flows the viscous stress in the fluid can be taken as zero. Hence it is assumed for the rest of this derivation that $\sigma^{f}=0$ and $\sigma^{s} \equiv \sigma$.

Substituting (13) into (12) and adding both phase equations to eliminate $\pi^{\beta}$ gives

$$
\nabla p=\nabla \cdot \sigma .
$$

Substituting the interaction term (14) into (12) gives

$$
\begin{aligned}
-\nabla\left(\phi^{s} p\right)+\nabla \cdot \sigma & =K\left(\mathbf{v}^{s}-v^{f}\right)-p \nabla \phi^{s}, \\
-\nabla\left(\phi^{f} p\right) & =-K\left(\mathbf{v}^{s}-v^{f}\right)+p \nabla \phi^{s} .
\end{aligned}
$$


Making use of $\phi^{s}=1-\phi^{f}$ leads to

$$
\begin{aligned}
\nabla \cdot \boldsymbol{\sigma} & =K\left(\mathbf{v}^{s}-\mathbf{v}^{f}\right)+\phi^{s} \nabla p, \\
\boldsymbol{0} & =-K\left(\mathbf{v}^{s}-\mathbf{v}^{f}\right)+\phi^{f} \nabla p .
\end{aligned}
$$

Elimination of $p$ from these equations yields

$$
\nabla \cdot \sigma=\frac{K}{\phi^{f}}\left(\mathbf{v}^{s}-v^{f}\right)
$$

The continuity equation can be rewritten as

$$
\nabla \cdot \mathbf{v}=0
$$

where $\mathbf{v}$ is the macroscopic medium velocity vector, $\mathbf{v}=\phi^{f} \mathbf{v}^{f}+\phi^{s} \mathbf{v}^{s}$. Using this in (20) gives

$$
\nabla p=\nabla \cdot \boldsymbol{\sigma}=\frac{1}{k}\left(\frac{\partial \mathbf{u}}{\partial t}-\mathbf{v}\right)
$$

where $k=\left(\phi^{f}\right)^{2} / K$ is the permeability, $\mathbf{u}$ is the displacement of the solid and $\partial \mathbf{u} / \partial t=\mathbf{v}^{s}$. A derivation of this relationship between $k$ and $K$ is given in [23].

Equation (22) can be easily explained in physical terms. Taking Darcy's law and expressing it relative to the movement of the solid it is easy to obtain $\nabla p=\frac{1}{k}(\partial \mathbf{u} / \partial t-\mathbf{v})$. Considering the stress in the solid matrix as being governed by the standard equilibrium equation of elasticity gives $\nabla \cdot \sigma=\nabla p$ where the gradient of pressure is acting as an internal body force on the solid matrix.

As a porous medium is compressed the resulting decrease in porosity will lead to a reduction in the permeability $k$. The simplest model that allows for this is if $k=k(\phi)$ where $\phi$ is the dilation (the change in porosity) of the medium. Various forms for this functional dependence have been considered $[23,22,3]$ and it seems that the most versatile is

$$
k=k_{0} \exp (m \phi)
$$

where $k_{0}$ and $m$ are constants.

\section{Governing equations}

In developing the governing equations it will be assumed that deformations are infinitesimal, that by symmetry only the radial components of displacement and velocity are nonzero and that the permeability function is dependent on the change in local porosity, $\phi$. 
The components of the stress tensor for the solid are

$$
\begin{aligned}
& \sigma_{r r}=(\lambda+2 \mu) \frac{\partial u}{\partial r}+n \lambda \frac{u}{r}, \\
& \sigma_{\theta \theta}=(\lambda+2 \mu) \frac{u}{r}+\lambda \frac{\partial u}{\partial r}+(n-1) \frac{u}{r} \lambda,
\end{aligned}
$$

where $n=0,1,2$ correspond to planar $(x, y, z)$, cylindrical $(r, \theta, z)$ and spherical $(r, \theta, \varphi)$ geometries respectively and $u \equiv u_{r}$, the radial displacement. Here $\lambda$ and $\mu$ are the Lamé stress constants. In the case of planar flow it should be remembered that $\sigma_{r r}$ is simply $\sigma$ with $r \equiv x$ and that the equation for $\sigma_{\theta \theta}$ does not apply. Also because of symmetry in the spherical case, $\sigma_{\theta \theta}=\sigma_{\varphi \varphi}$. All other stress components are zero. The divergence of the stress in the radial direction can be written as

$$
(\nabla \cdot \sigma)_{r}=\frac{\partial \sigma_{r r}}{\partial r}+n \frac{\sigma_{r r}-\sigma_{\theta \theta}}{r} .
$$

It can also be shown, with a little algebra, that substitution of (24) and (25) into (26) gives

$$
(\nabla \cdot \sigma)_{r}=H_{a} \frac{\partial \phi}{\partial r}
$$

where

$$
\phi=\frac{1}{r^{n}} \frac{\partial}{\partial r}\left(r^{n} u\right)
$$

is the local change in porosity and $H_{a}=\lambda+2 \mu$ is the aggregate modulus. Integration of the continuity equation (21) leads to the macroscopic velocity component in the radial direction

$$
v_{r}=\frac{v(t)}{r^{n}}
$$

The governing equation (22) then becomes

$$
\frac{\partial p}{\partial r}=H_{a} \frac{\partial}{\partial r}\left(\frac{1}{r^{n}} \frac{\partial}{\partial r}\left(r^{n} u\right)\right)=\frac{1}{k(\phi)}\left(\frac{\partial u}{\partial t}-\frac{v(t)}{r^{n}}\right) .
$$

A useful transformation

$$
w(r, t)=-u(r, t)+\frac{1}{r^{n}} \int_{0}^{t} v(\tau) d \tau
$$

allows the governing equation to be written as

$$
\frac{\partial}{\partial r}(L[w])=\frac{1}{k(L[w])} \frac{\partial w}{\partial t},
$$

where the operator $L$ is given by

$$
L[w]=\frac{1}{r^{n}} \frac{\partial}{\partial r}\left(r^{n} w\right)
$$


The velocity term has now been removed from the governing equation. Alternatively equations in terms of $\phi$ can be derived. If (30) is rewritten using (28) as

$$
H_{a} k(\phi) \frac{\partial \phi}{\partial r}=\frac{\partial u}{\partial t}-\frac{v(t)}{r^{n}}
$$

and the operator $L$ applied to both sides, the governing equation becomes

$$
H_{a} \frac{1}{r^{n}} \frac{\partial}{\partial r}\left(k(\phi) r^{n} \frac{\partial \phi}{\partial r}\right)=\frac{\partial \phi}{\partial t}
$$

which is also independent of the velocity. It will be shown in the next section that the boundary conditions for (35) can also be expressed independent of the velocity. This allows solutions to be found for the more experimentally feasible case of a specified pressure gradient rather than a specified velocity. Depending on the situation, either (30), (32) or (35) can be used to evaluate the displacement. Equation (35) is preferable if the pressure is specified, while (32) would be more suitable if the velocity is given.

\section{Boundary conditions}

The problem under consideration is shown in Figure 1. A section of a cylinder or a sphere is shown with fluid moving radially out from the centre. The outer boundary is constrained by a rigid mesh that offers no resistance to the passage of the fluid. The inner radius is $a$ and the outer radius is $b$.

The boundary condition for the displacement at the rigid porous mesh, $r=b$, is simply $u=0$. Making use of the relation for $\phi$ in (28) this can

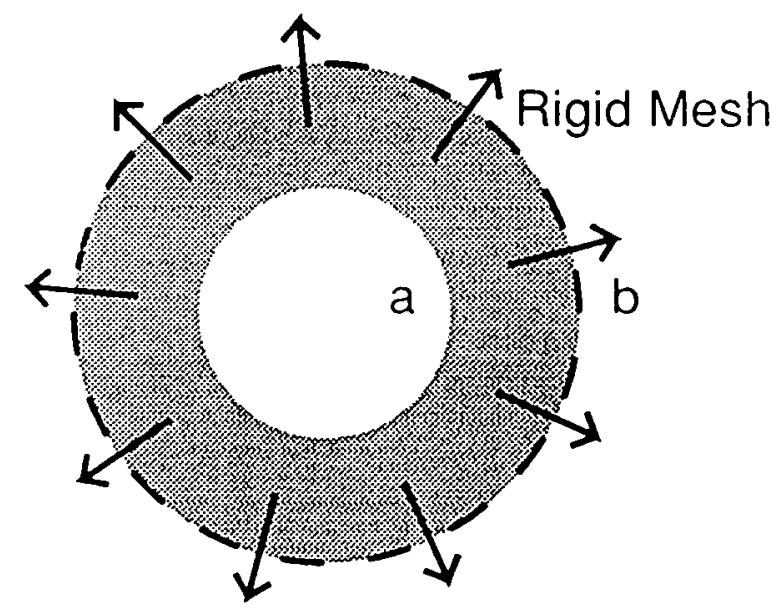

FIGURE 1. Schematic diagram of radial flow through a cylindrical or a spherical shell. In this case of constrained flow the outer boundary is constrained by a rigid mesh. 
also be written as

$$
\phi(b, t)=\frac{\partial u}{\partial r}(b, t)
$$

At the free boundary, $r=a$, the contact stress is zero since the flow is normal to the surface [12] and so

$$
\sigma_{r r}(a, t)=0
$$

This can be written as

$$
\left[\frac{\partial u}{\partial r}+n \bar{\lambda} \frac{u}{r}\right]_{r=a}=0
$$

where $\bar{\lambda}=\lambda /(\lambda+2 \mu)$. In terms of $\phi$ this is

$$
\phi(a, t)=n \frac{u(a, t)}{a}(1-\bar{\lambda}) \text {. }
$$

By integrating (28) the displacement can be written as

$$
u(r, t)=-\frac{1}{r^{n}} \int_{r}^{b} \alpha^{n} \phi(\alpha, t) d \alpha .
$$

By substitution of $u$ into (39) a boundary condition in terms of $\phi$ is derived as

$$
\phi(a, t)+n \frac{(1-\bar{\lambda})}{a^{n+1}} \int_{0}^{b} \alpha^{n} \phi(\alpha, t) d \alpha=0 .
$$

Making use of the relation between pressure and stress (30), the boundary condition at the constrained boundary, $r=b$, is

$$
\phi(b, t)=\phi(a, t)-\frac{\Delta P(t)}{H_{a}} .
$$

Equations (41) and (42) then form boundary conditions for (35). Boundary conditions for the variable $w$ in (31) can be written simply as

$$
\begin{aligned}
w(b, t) & =-\frac{1}{b^{n}}-\int_{0}^{t} v(\tau) d \tau, \\
{\left[\frac{\partial w}{\partial r}+n \frac{w}{r} \bar{\lambda}\right]_{r=a} } & =\frac{1}{a^{n+1}}(\bar{\lambda}-1) \int_{0}^{t} v(\tau) d \tau .
\end{aligned}
$$

The problem of the position of the inner boundary, $a$, is a potentially important one usually avoided. It can be shown [4] that the intuitive result

$$
a=a^{0}+u(a, t),
$$

is valid where the original position of the boundary is denoted $a^{0}$. This is an implicit equation for the new boundary position, $a$. It can only be solved after the displacement function has been evaluated and will normally require a numerical solution. 
An approximate form would be to assume the explicit relation

$$
a=a^{0}+u\left(a^{0}, t\right),
$$

where the boundary condition is applied at the initial position of the boundary. The error associated with this approximation is discussed in the next section.

Alternatively a Lagrangian coordinate frame can be considered ([3], [5]) allowing the boundary conditions to be applied at the initial boundary position as in (45). The transformation from Eulerian to Lagrangian frameworks, however, adds further nonlinearities to the governing equation (35). For large deformations, nonlinear stress relations are required ([3], [5]) and the Lagrangian formulation would be preferred. For small deformations it is often advantageous to use Eulerian coordinates, hence keeping the governing equation simple, at the expense of having a nonlinear boundary condition (44).

\section{Steady radial flow}

The governing equation and boundary conditions are nondimensionalised using

$$
\begin{gathered}
p=p_{m} \hat{p}, \quad r=b \hat{r}, \quad k=k_{0} \hat{k}, \\
u=u_{0} \hat{u}, \quad v=-\hat{v} H_{a} k_{0} b^{n} \frac{u_{0}}{b}, \quad a=b \hat{a},
\end{gathered}
$$

for typical pressure $p_{m}$, outer radius $b$, permeability $k_{0}$ and displacement $u_{0}$. The steady form of the governing equation (30) can then be written as

$$
B \frac{d \hat{p}}{d \hat{r}}=\frac{d \hat{\phi}}{d \hat{r}}=\frac{\hat{v}}{\hat{r}^{n} \hat{k}(\delta \hat{\phi})},
$$

where

$$
\hat{\phi}=\frac{1}{\hat{r}^{n}} \frac{d}{d \hat{r}}\left(\hat{r}^{n} \hat{u}\right)
$$

and dimensionless parameters are

$$
\delta=\frac{u_{0}}{b}, \quad B=\frac{p_{m}}{H_{a}} \frac{1}{\delta} .
$$

The hats denoting nondimensional variables will now be dropped as all variables will be assumed nondimensional unless stated otherwise.

The parameter $\delta$ is now taken to be $\delta=1$, equivalent to nondimensionalising $u$ with respect to $b$. In the next section this parameter will be 
reintroduced in a perturbation analysis. By using the function

$$
g(\phi)=\int k(\phi) d \phi,
$$

the governing equation (47) can be written as

$$
\frac{d}{d r}[g(\phi)]=\frac{v}{r^{n}} .
$$

Integration yields

$$
g(\phi)= \begin{cases}(1-n) v r^{1-n}+c_{1} & \text { for } n=0 \text { or } 2, \\ v \ln r+c_{1} & \text { for } n=1,\end{cases}
$$

where $c_{1}$ is a constant of integration. Inverting this equation, assuming that $g^{-1}$ exists, yields

$$
\frac{1}{r^{n}} \frac{d}{d r}\left(r^{n} u\right)= \begin{cases}g^{-1}\left((1-n) v r^{1-n}+c_{1}\right) & \text { for } n=0 \text { or } 2, \\ g^{-1}\left(v \ln r+c_{1}\right) & \text { for } n=1 .\end{cases}
$$

This can be integrated to obtain

$$
u(r)= \begin{cases}-\frac{1}{r^{n}} \int_{r}^{1} s^{n} g^{-1}\left((1-n) v s^{1-n}+c_{1}\right) d s+\frac{c_{2}}{r^{n}} & \text { for } n=0 \text { or } 2, \\ -\frac{1}{r} \int_{r}^{1} s g^{-1}\left(v \ln s+c_{1}\right) d s+\frac{c_{2}}{r} & \text { for } n=1,\end{cases}
$$

where $c_{2}$ is a constant of integration. Using the boundary condition of $u(1)=0$ it is easy to see that $c_{2}=0$. At $r=a$, the inner boundary,

$$
\phi(a)=\frac{n u(a)(1-\bar{\lambda})}{a} .
$$

Also from (52)

$$
c_{1}= \begin{cases}g[\phi(a)]-(1-n) v a^{1-n} & \text { for } n=0,2, \\ g[\phi(a)]-v \ln a, & \text { for } n=1\end{cases}
$$

Substituting this into (54) gives

$$
u(r)=-\frac{1}{r^{n}} \int_{r}^{1} s^{n} g^{-1}\left((1-n) v\left[s^{1-n}-a^{1-n}\right]+g\left[\frac{n u(a)(1-\bar{\lambda})}{a}\right]\right) d s,
$$

for $n=0$ or 2 , and

$$
u(r)=-\frac{1}{r} \int_{r}^{1} s g^{-1}\left(v \ln \left[\frac{s}{a}\right]+g\left[\frac{u(a)}{a}(1-\bar{\lambda})\right]\right) d s,
$$

for $n=1$. The pressure can be found from (42) which gives

$$
\frac{\Delta P}{H_{a}}=n \frac{u(a)}{a}(1-\bar{\lambda})-\left.\frac{d u}{d r}\right|_{r=1} \text {. }
$$


The derivative $d u / d r$ can be expressed in terms of the inner radius $a$ using the relationships

$$
\begin{array}{ll}
\quad g\left(\left.\frac{d u}{d r}\right|_{r=1}\right)=g\left(\frac{u(a)}{a}(1-\bar{\lambda})\right)-v \ln a, & n=1, \\
g\left(\left.\frac{d u}{d r}\right|_{r=1}\right)-(1-n) v(1)^{1-n} & =g\left(\frac{n u(a)}{a}(1-\bar{\lambda})\right)-(1-n) v(a)^{1-n}, \quad n=0,2,
\end{array}
$$

which are found by expressing the constant $c_{1}$ in (53) in terms of both the inner and outer boundary conditions.

Thus if the permeability function is known and (57) and (58) are analytically integrable then an implicit equation for $a$ is found which can be solved numerically, and the pressure can then be found directly.

When solving the governing equation, many authors apply the boundary conditions at the original positions of the boundary $a^{0}$ even though using Eulerian coordinates. The assumption, often not stated, is that the error in applying the boundary conditions at $a^{0}$ rather than at $a$ will be negligible for sufficiently small deformations. A nonlinear permeability relation is often used to provide more accurate results even though the boundary conditions are still approximate. The relative error of the boundary assumption versus the change due to permeability relation is now investigated.

Using a small nondimensional velocity of $v=-0.1$, parameter $\bar{\lambda}=0.6$ and initial inner radius of 0.5 we calculate the displacement for flow in a cylindrical shell for three situations. For case 1, we use using a constant permeability relation, $k=1$, with the inner radii $a$ found numerically using the exact equation (44). Equation (58) was used to evaluate $u(r)$. For case 2 , we consider a constant permeability where the approximate boundary condition (45) is used. For case 3 , we consider a permeability relation $k=$ $1 /(1-m \phi), m=4.0$ with the exact boundary condition, (44), used. This form for the permeability was chosen since it yields exact integral results via equation (58) and has been used previously for arterial tissue [22]. These are depicted in Figure 2. The solid line indicates case 1, the dashed line case 2 , and the dotted line case 3 . The maximum displacements shown here are approximately $2 \%$ of the outer radius. We also note that when $\bar{\lambda}$ is varied the relative order of the solutions (case $2<$ case $1<$ case 3 ) changes.

The displacements show that the error is using an approximate boundary condition is of the same order of magnitude as the improvement in the model by using a nonlinear permeability. Thus a nonlinear permeability relation should only be used if the boundary conditions are also applied correctly. If analytical solutions are required for more complicated geometries the fully 


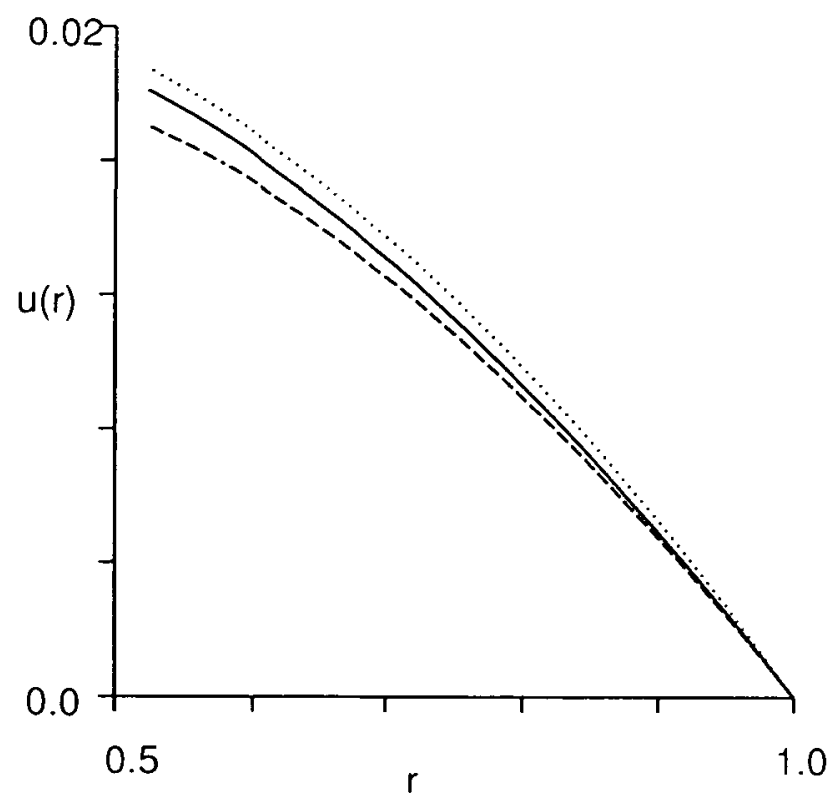

FIGURE 2. Equilibrium displacement, $u(r)$, for a cylindrical shell with $0.5 \leq r \leq 1$, $\bar{\lambda}=0.6, v=-0.1$ and a constrained outer boundary. Solid line: Solution with $k=1$ and exact boundary position; Dotted line: Solution with $k=1 /(1-4 \phi)$ and exact boundary position; Dashed line: Solution with $k=1$ and boundary conditions applied at the initial boundary positions.

linearised problem (case 2) can be solved as long as both sources of error are recognized as limitations on the solution.

The change in porosity $\phi$ is shown for cylindrical and planar geometries in Figure 3. This illustrates that although in the planar case the medium is always under compression, the cylindrical porous shell undergoes an expansion in the inner regions of the shell. This is due to annular stretching of the medium as the inner region of the shell increases in radius. The annular expansion more than compensates for the radial contraction $d u / d r$ in the inner region. It is apparent that the planar flow leads to finer filtration for the same applied pressure, filtering a smaller particle size in the highly compressed downstream region. However in some applications of filters, particularly in ultrafiltration of organic compounds, the aim is to capture the macromolecules within the porous medium to be recovered after the flux is stopped. The cylindrical shell will then be able to 'capture' larger molecules than the planar geometry in which larger particles are excluded from the medium. Average sized particles will also be able to travel further into the medium before becoming trapped, hence increasing the probability that they will remain permanently trapped when the fluid flux is stopped. 


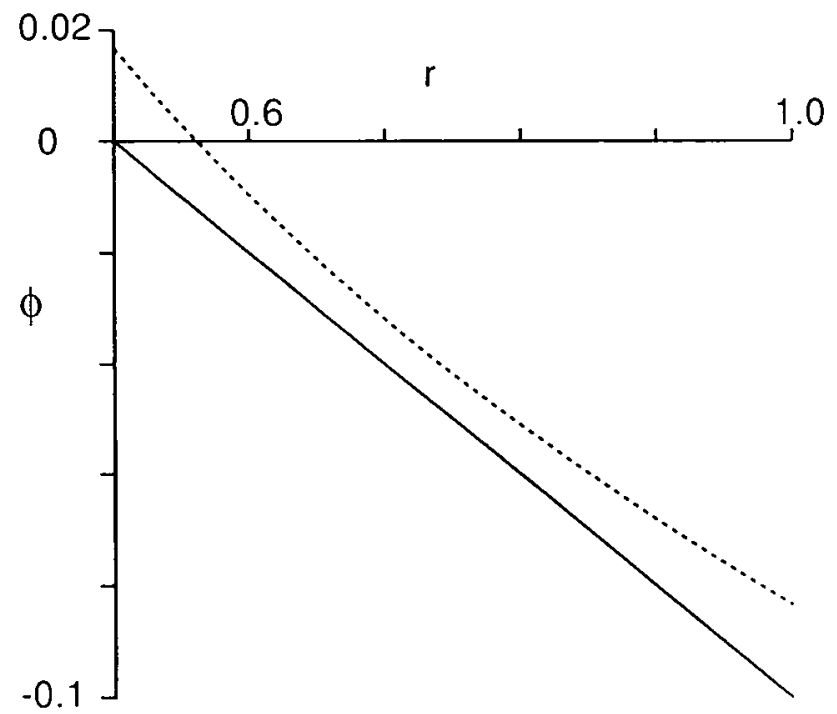

FIGURE 3. The change in porosity $\phi$ for planar (solid line) and cylindrical (dashed line) problems. The applied pressure was $\Delta P / H_{a}=0.1$ and $\bar{\lambda}=0.5$. Note the expansion in the inner region of the cylindrical shell.

The expansion of the inner section of the medium for small times can play an important role in the dynamics of the solid-fluid interaction. Firstly care must be taken in choosing a permeability relation that gives feasible values for an expanded medium. Secondly it is as yet unknown whether for some media the permeability should be a function of the porosity or a function of the radial strain $d u / d r$. For random media the permeability dependence on porosity is probably correct.

\section{Perturbation solution for infinitesimal displacements}

When analytic solutions via (57) and (58) are not possible, a perturbation technique can be employed. The parameter $\delta$ is now reintroduced as a perturbation parameter assuming that the deformation is small compared to the outer radius of the shell. The permeability can then be written in the form

$$
\frac{1}{k(\delta \phi)}=\zeta(\delta \phi)=1+\delta \zeta^{\prime}(0) \phi+\delta^{2} \zeta^{\prime \prime}(0) \frac{\phi^{2}}{2}+O\left(\delta^{3}\right) .
$$

In the limit of no deformation, $k(\phi)=1$ as $\phi \rightarrow 0$ and therefore $\zeta(0)=1$. 
By defining the displacement as

$$
u(r)=u_{0}(r)+\delta u_{1}(r)+\delta^{2} u_{2}(r)+O\left(\delta^{3}\right),
$$

the governing equation (47) can be expanded in powers of $\delta$. Taking coefficients of $\delta$, the zeroth order equation is

$$
r^{n}\left(\frac{d^{2} u_{0}}{d r^{2}}+\frac{n}{r} \frac{d u_{0}}{d r}-\frac{n}{r^{2}} u_{0}\right)=v
$$

which has solutions

$$
u_{0}(r)= \begin{cases}c_{3} r+\frac{c_{4}}{r^{2}}-\frac{v}{2}, & \text { for } n=2, \\ c_{3} r+\frac{c_{4}}{r}+v\left(\frac{r}{2} \ln r-\frac{r}{4}\right), & n=1, \\ c_{3} r+c_{4}+r^{2} \frac{v}{2}, & n=0,\end{cases}
$$

where $c_{3}, c_{4}$ are constants of integration found from application of the boundary conditions. The first order equation then becomes

$$
r^{n}\left(\frac{d^{2} u_{1}}{d r^{2}}+\frac{n}{r} \frac{d u_{1}}{d r}-\frac{n}{r^{2}} u_{1}\right)=v \zeta^{\prime}(0)\left(\frac{d u_{0}}{d r}+\frac{n}{r} u_{0}\right),
$$

which can be easily integrated using standard algebraic manipulation routines.

\section{Small time solutions in unsteady flow}

Upon application of a pressure gradient the initial behaviour of the porous medium is governed by the linearised form of (35) using a constant permeability. The initial deformation is not large enough to change the permeability significantly. The governing equation can then be written as

$$
\frac{1}{r^{n}} \frac{\partial}{\partial r}\left(r^{n} \frac{\partial \phi}{\partial r}\right)=\frac{\partial \phi}{\partial t}
$$

with boundary and initial conditions

$$
\begin{aligned}
& \phi(a, t)=-(1-\bar{\lambda}) \frac{n}{a^{n+1}} \int_{a}^{1} \alpha^{n} \phi(\alpha, t) d \alpha, \\
& \phi(1, t)=\phi(a, t)-\Delta P(t), \\
& \phi(r, 0)=0 .
\end{aligned}
$$

Here we assume that the boundary conditions are applied at $r=a$ rather than at $r=a(t)$, an approximation that is justified since we are using a linear infinitesimal theory valid only for small times when the medium has 
not deformed far from the initial position. The nonlinear moving boundary problem is currently the subject of further research. Solutions to the linear problem can be found using a number of approaches. For oscillatory pressure gradients the Fourier components of the system could be found. As the governing equation here is only valid for the infinitesimal displacements present in the initial stages of the deformation a Laplace transform technique is preferred. Taking the Laplace transform of (66) gives

$$
r^{2} \bar{\phi}^{\prime \prime}+n r \bar{\phi}^{\prime}-s \bar{\phi} r^{2}=0
$$

where $\mathscr{L}\{\phi(r, t)\}=\bar{\phi}(r, s)$ and $\bar{\phi}^{\prime}=\partial \bar{\phi}(r, s) / \partial r$. Using $\eta=r \sqrt{s}$ this can be written as

$$
\eta^{2} \bar{\phi}^{\prime \prime}+n \eta \bar{\phi}^{\prime}-\eta^{2} \bar{\phi}=0
$$

where now $\bar{\phi}^{\prime}=\partial \bar{\phi}(\eta, s) / \partial \eta$. The solution of (69) in the case of cylindrical geometry is

$$
\bar{\phi}(\eta, s)=A_{1}(s) I_{0}(\eta)+A_{2}(s) K_{0}(\eta),
$$

where $I_{0}, K_{0}$ are modified Bessel functions of order zero and $A_{1}, A_{2}$ are functions found from application of the boundary conditions to be

$$
\begin{aligned}
A_{1}(s) & =\xi(s) A_{2}(s), \\
A_{2}(s) & =\frac{\overline{\Delta P}}{K_{0}\left(\eta_{a}\right)-K_{0}\left(\eta_{b}\right)-\xi(s)\left(I_{0}\left(\eta_{a}\right)-I_{0}\left(\eta_{b}\right)\right)}, \\
\xi(s) & =\frac{\eta_{a}^{2} K_{0}\left(\eta_{a}\right)-\hat{\lambda}\left(-\eta_{a} K_{1}\left(\eta_{a}\right)+\eta_{b} K_{1}\left(\eta_{b}\right)\right)}{\eta_{a}^{2} I_{0}\left(\eta_{a}\right)-\hat{\lambda}\left(\eta_{a} I_{1}\left(\eta_{a}\right)-\eta_{b} I_{1}\left(\eta_{b}\right)\right)},
\end{aligned}
$$

where $\eta_{a}=a \sqrt{s}, \eta_{b}=b \sqrt{s}$ and $\hat{\lambda}=1-\bar{\lambda}$. Abramowitz and Stegun [1, equations 11.3.25-11.3.28] was used to evaluate the integrals of the Bessel functions. This is difficult to invert in general. However, a small-time solution can be found by inverting the asymptotic form valid for $s \rightarrow \infty$ of the above solution. Using the asymptotic results for $I_{\nu}, K_{\nu}$ from Abramowitz and Stegun [1, equations 9.7.1 and 9.7.2] it can be shown that as $s \rightarrow \infty$

$$
\begin{aligned}
\xi(s) & \sim \frac{\eta_{a}^{2} K_{0}\left(\eta_{a}\right)+\hat{\lambda} \eta_{a} K_{1}\left(\eta_{a}\right)}{\hat{\lambda} \eta_{b} I_{1}\left(\eta_{b}\right)} \\
& \sim e^{-\eta_{a}-\eta_{b}} \sqrt{\frac{a}{b}} \frac{\pi}{\hat{\lambda}} \eta_{a}\left(1+\frac{c_{1}}{\eta_{a}}+\frac{c_{2}}{\eta_{a}^{2}}\right) .
\end{aligned}
$$

Since $a<b$ and $s \rightarrow \infty$ then $\eta_{a} \rightarrow \infty$ so $e^{\eta_{a}} \ll e^{\eta_{b}}$. It is easy to see that $\eta_{b}=\eta_{a} b / a$. The constants $c_{1}, c_{2}$ were determined using Mathematica [30] 


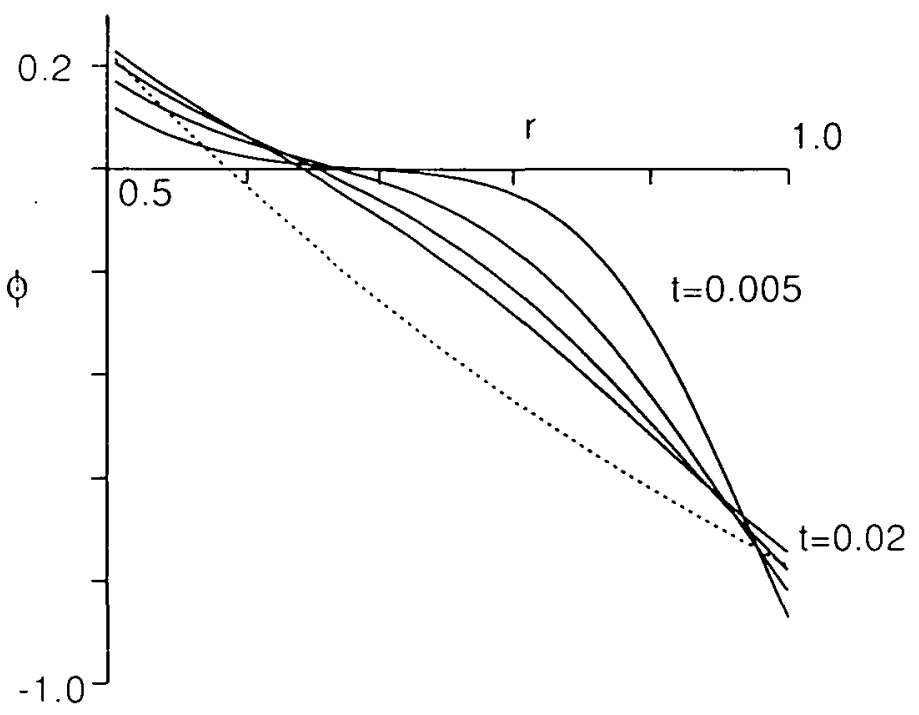

FIGURE 4. Change the porosity, $\phi(r)$, for a constrained cylindrical shell $0.5 \leq r \leq 1$. The solid lines are $\phi$ at four small times, $t=0.005,0.01,0.015,0.02$. The dotted line is the final equilibrium state of the shell.

and are

$$
\begin{aligned}
& c_{1}=-\frac{1}{8}+\frac{3 a}{8 b}+\hat{\lambda}, \\
& c_{2}=\frac{9}{128}+\frac{33}{128} \frac{a^{2}}{b^{2}}+\frac{3 \hat{\lambda}}{8}+\frac{3 a}{8 b} \hat{\lambda} .
\end{aligned}
$$

Also

$$
\left[K_{0}\left(\eta_{a}\right)-K_{0}\left(\eta_{b}\right)-\xi(s)\left(I_{0}\left(\eta_{a}\right)-I_{0}\left(\eta_{b}\right)\right)\right]^{-1} \approx \sqrt{\frac{2}{\pi}} \frac{b}{a} \hat{\lambda} \frac{e^{\eta_{a}}}{\sqrt{\eta_{a}}}\left(1+\frac{c_{3}}{\eta_{a}}\right),
$$

where

$$
c_{3}=-\frac{1}{8}+\frac{a}{2 b}+\hat{\lambda}+\frac{b \hat{\lambda}}{a} .
$$

The constants $A_{1}, A_{2}$ can then be determined giving the final solution as

$$
\bar{\phi} \approx \overline{\Delta P} e^{-(r-a) \sqrt{s}} \hat{\lambda} \frac{b}{a} \frac{1}{\sqrt{r a s}}-\overline{\Delta P} e^{-(b-r) \sqrt{s}} \sqrt{\frac{b}{r}}\left(1+\frac{c_{4}(r)}{a \sqrt{s}}\right) .
$$

The "constant"

$$
c_{4}(r)=-\left(\frac{a}{8 b}+\frac{b \hat{\lambda}}{a}-\frac{a}{b r}\right)
$$

was determined using Mathematica [30] and is needed in the solution to ensure both parts are given to the same order of accuracy. For an applied 


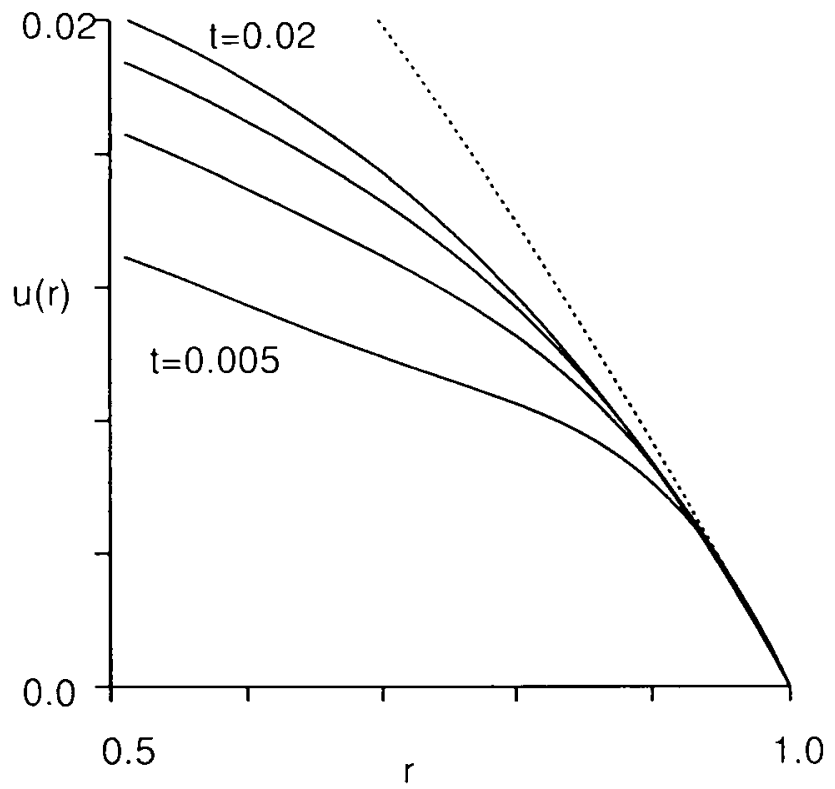

FIGURE 5. Displacement, $u(r)$, for a constrained cylindrical shell with $0.5 \leq r \leq 1$. The solid lines are $u$ at four times, $t=0.005,0.01,0.015,0.02$. The dotted line is the final equilibrium state of the shell. Note the formation of a maximum consolidation region near $r=1$ that expands inwards with time.

pressure gradient of the form $\Delta P=t^{m / 2}, m=0,1,2, \ldots$, the solution then becomes as $t \rightarrow 0$

$$
\begin{aligned}
\phi(r, t) & \rightarrow \Gamma\left(1+\frac{m}{2}\right)(1-\bar{\lambda}) \frac{b}{a \sqrt{r a}}(4 t)^{(m+1) / 2} \mathrm{i}^{m+1} \operatorname{erfc}\left(\frac{r-a}{2 \sqrt{t}}\right) \\
- & \Gamma\left(1+\frac{m}{2}\right) \sqrt{\frac{b}{r}}(4 t)^{m / 2}\left[\mathrm{i}^{m} \operatorname{erfc}\left(\frac{b-r}{2 \sqrt{t}}\right)+2 \sqrt{t} \frac{c_{4}(r)}{a} \mathrm{i}^{m+1} \operatorname{erfc}\left(\frac{b-r}{2 \sqrt{t}}\right)\right] .
\end{aligned}
$$

The notation ierfc denotes integration of the complementary error function. The displacement is found from numerical integration of $\phi$. Although the full time dependent behaviour of radial flow may be obtained using a numerical scheme, much of the useful information of the deformation can be found analytically from the small-time behaviour of the displacement. Figure 4 shows the porosity change $\phi$ at four different times after application of a unit step change in pressure. The inner radius was initially chosen to be 0.5 . The final equilibrium result is shown as the dotted curve and is found from solving the steady-state equations.

We note that the expansion of the medium at the inner boundary increases with time. The results also show that a highly compressed region forms at the constrained boundary and spreads inwards as time increases. Figure 5 


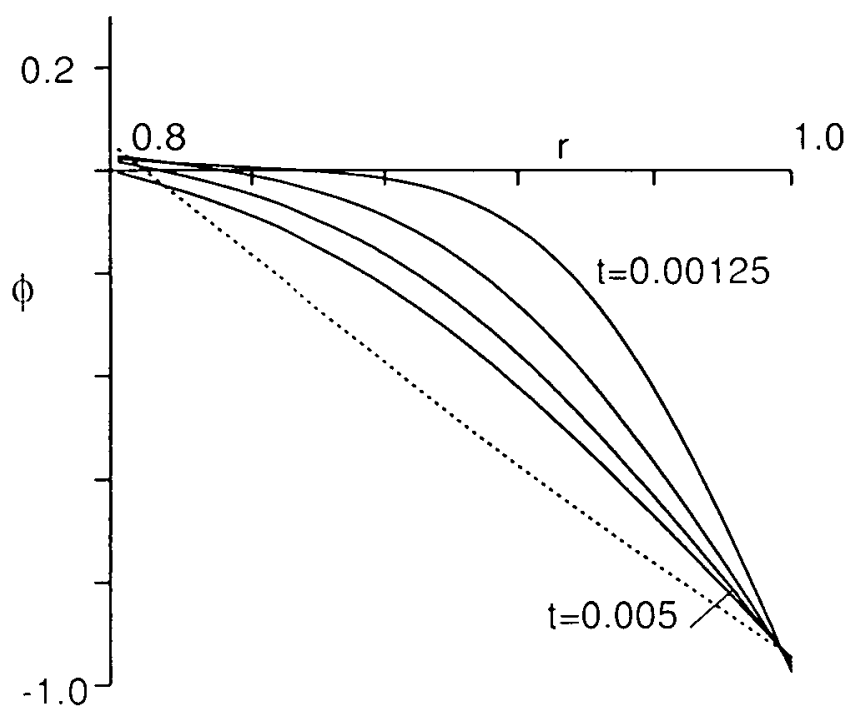

Figure 6. Change in porosity, $\phi(r)$, for a constrained cylindrical shell $0.8 \leq r \leq 1$. The solid lines are $\phi$ at four small times, $t=0.0025,0.0375,0.005$. The dotted line is the final equilibrium state of the shell. Note that very little local expansion occurs with this thin shell.

shows the displacement as a function of radial distance for various times, $t=0.005,0.01,0.015,0.02$, confirming the existence of a consolidation region growing in time. A maximum displacement gradient is reached at $r=$ 1.0 representing the limit of consolidation. In Figure 6 the porosity and the displacement are shown for the case of a thin shell where $0.8 \leq r \leq 1$. Since the thin-walled shell reaches equilibrium faster than the thick-walled shell the maximum time plotted is $t=0.005$. Note that there is little expansion of the inner region of the shell. Uniform results should be expected since the shell is essentially thin enough to react like a uniform medium for most values of time.

\section{Perturbation solutions for slow compression rates}

The governing equation for this case from (35) is

$$
\frac{1}{r^{n}} \frac{\partial}{\partial r}\left(k(\delta \phi) r^{n} \frac{\partial \phi}{\partial r}\right)=\frac{1}{R^{2}} \frac{\partial \phi}{\partial t}
$$

where $R^{2}=t_{0} H_{a} k_{0} / b^{2}$ and boundary conditions are given by (67). Since the compression is slow, the parameter $1 / R^{2} \ll 1$ and the problem can be 
expressed as a power series

$$
\phi(r, t)=\frac{1}{R^{2}} \phi_{1}(r, t)+\frac{1}{R^{4}} \phi_{2}(r, t)+\cdots .
$$

The permeability is expanded in the form

$$
k(\delta \phi) \approx 1+\frac{1}{R^{2}}\left(\delta k^{\prime}(0) \phi_{1}\right)+\frac{1}{R^{4}}\left(\delta k^{\prime}(0) \phi_{2}+\delta^{2} k^{\prime \prime}(0) \phi_{1}^{2}\right)
$$

The coefficient of the first order of $1 / R^{2}$ is the steady-state equation

$$
\frac{1}{r^{n}} \frac{\partial}{\partial r}\left(r^{n} \frac{\partial \phi_{1}}{\partial r}\right)=0
$$

giving

$$
\phi_{1}(r, t)= \begin{cases}\frac{c_{1}(t)}{r}+c_{2}(t), & \text { for } n=2, \\ c_{1}(t) \ln (r)+c_{2}(t), & \text { for } n=1, \\ c_{1}(t) r+c_{2}(t), & \text { for } n=0,\end{cases}
$$

where $c_{1}(t)$ and $c_{2}(t)$ are constants of integration. The equation for the second-order $\phi_{2}(r, t)$ is

$$
\frac{1}{r^{n}} \frac{\partial}{\partial r}\left(r^{n} \frac{\partial \phi_{2}}{\partial r}\right)=-\delta k^{\prime}(0) \frac{1}{r^{n}} \frac{\partial}{\partial r}\left(r^{n} \phi_{1} \frac{\partial \phi_{1}}{\partial r}\right)+\frac{\partial \phi_{1}}{\partial t},
$$

which can be solved easily by successive integrations.

The first-order solution can be seen to be the solution to the linearised time independent equation. Thus $\phi$ behaves as a succession of steady states with the higher-order terms incorporating nonlinear effects. This has been discussed in some depth in Barry and Aldis [5] for the planar case.

Since the parameter $1 / R^{2}$ is small, we note that the time derivative disappears in the limit of $1 / R^{2} \rightarrow 0$, hence making the governing equation (83) singular in time. This can be overcome by redefining a new time variable $\tau=R^{2} t$ which will allow us to obtain solutions for small times. A perturbation expansion in $1 / R^{2}$ then gives $(66)$ for the first-order equation. That is, for small-times we expect the equation to be linear since the permeability is essentially unchanged.

Figure 7 is a schematic diagram of the deformation of the inner boundary against time under the action of an applied pressure gradient $\Delta P=t$. Initially the medium is not highly compressed and the small-time linear solution is valid. For later times the solution acts as a sequence of steady states with a time lag due to higher-order terms, and then the nonlinear effects become apparent. 


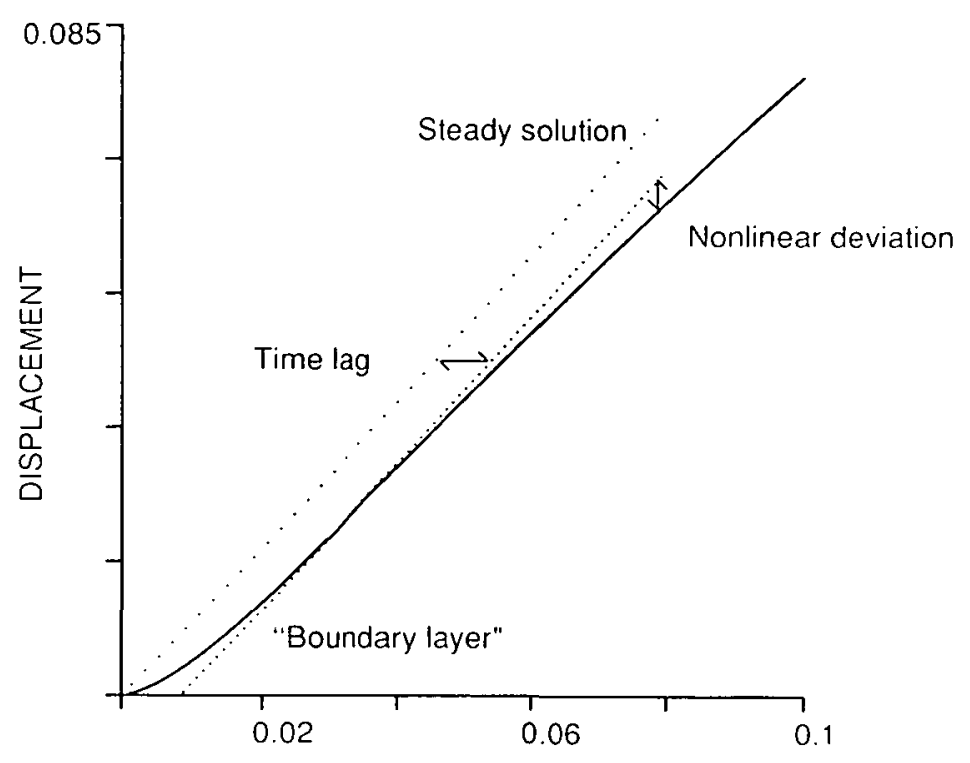

TIME

FIGURE 7. Schematic diagram of the displacement of the inner boundary versus time for an applied pressure $\Delta P=t$. A small boundary layer is followed by the solution for a succession of steady states with time lag, and then nonlinear effects become important.

\section{Conclusions}

The theory of mixtures has been applied to flow through deformable porous media to develop general nonlinear equations and boundary conditions governing the radially directed flow through either spherical, cylindrical or planar shells. General solutions for steady flow were given as integrals involving the permeability function. In addition, a perturbation solution valid for typical permeability functions was given. For unsteady flow an approximate solution valid for small time was given as well as solutions valid for slow compression rates. Figures were presented comparing flow in planar and cylindrical geometries.

In planar geometries, the medium becomes more compressed for a given pressure than the cylindrical medium, thus filtering out smaller particles. The change in porosity was also shown to decrease in the planar case, but for cylindrical shells of sufficient width, a significant region of expanded medium occurs. Thus a cylindrical filter will trap within its expanded pores a larger sized particle for later recovery. It was also shown that error associated with use of approximated boundary conditions can negate the increased accuracy with use of nonlinear permeability relations. 


\section{Acknowledgements}

This research was partially undertaken at the Physiological Flow Studies Unit, Imperial College, London while S. Barry was on a British Council Postgraduate Bursary. S. Barry would like to thank Dr. K. Parker and Dr. J. Lever for interesting discussions. This work was completed with the assistance of an A.R.C. grant.

\section{References}

[1] M. Abramowitz and I. A. Stegun, Handbook of Mathematical Functions, (Dover, New York, 1972).

[2] R. J. Atkin and R. E. Craine, "Continuum theories of mixtures: Basic theory and historical development", Quart. J. Mech. Appl. Math. 29 (1976) 209-244.

[3] S. I. Barry and G. K. Aldis, "Comparison of models for flow induced deformation of soft biological tissue", J. Biomechanics 23 (1990) 647-654.

[4] S. I. Barry, "Flow in a deformable porous medium", Ph.D. Thesis, University College, University of New South Wales, 1990.

[5] S. I. Barry and G. K. Aldis, "Unsteady flow induced deformation of porous materials", Int. J. Non-Linear Mech. 26 (1991) 687-699.

[6] N. Barton, I. Howells (moderators), "Some Mathematical Aspects of Hollow Fibre U1trafiltration", in Proc. 1986 Math. in Industry Study Group, (ed: F. De Hoog) (CSIRO Div. Math. Stats., Australia) (1987) 1-21.

[7] A. Bedford and D. S. Drumheller, "Recent advances, theory of immiscible and structured mixtures", Int. J. Engng. Sci. 21 (1983) 863-960.

[8] M. A. Biot, "General theory of three-dimensional consolidation", J. Appl. Phys. 12 (1941) 155-164.

[9] M. A. Biot, "Theory of elasticity and consolidation for a porous anisotropic solid", $J$. Appl. Phys. 26 (1955) 182-185.

[10] M. A. Biot, "Mechanics of deformation and acoustic propagation in porous media", $J$. Appl. Phys. 27 (1956) 1482-1498.

[11] R. M. Bowen, "Incompressible porous media models by the theory of mixtures", Int. J. Engng. Sci. 18 (1980) 1129-1148.

[12] J. S. Hou, M. H. Holmes, W. M. Lai and V. C. Mow, "Boundary conditions at the cartilage-synovial fluid interface for joint lubrication and theoretical verifications", $J$. Biomech. Engng. 111 (1989) 78-87.

[13] M. H. Holmes, "A nonlinear diffusion equation arising in the study of soft tissue”, Quart. Appl. Math. 61 (1983) 209-220.

[14] M. H. Holmes, "Comparison theorems and similarity solution approximation for a nonlinear diffusion equation arising in the study of soft tissue", SIAM J. Appl. Math. 44 (1984) 545-556.

[15] M. H. Holmes, "Finite deformation of soft tissue: analysis of a mixture model in uniaxial compression", J. Biomech Engng. 108 (1986) 372-381.

[16] R. Jain and G. Jayaraman, "A theoretical model for water flux through the arterial wall", J. Biomech. Engng. 109 (1987) 311-317.

[17] G. Jayaraman, "Water transport in the arterial wall-a theoretical study", J. Biomechanics 16 (1983) 833-840.

[18] D. E. Kenyon, "The theory of an incompressible solid-fluid mixture", Arch. Rat. Mech. Anal. 62 (1976) 131-147. 
[19] D. E. Kenyon, “Transient filtration in a porous elastic cylinder”, J. Appl. Mech. 98 (1976) 594-598.

[20] D. E. Kenyon, “Consolidation in compressible mixtures”, J. Appl. Mech. 45 (1978) $727-$ 732.

[21] D. E. Kenyon, “A mathematical model of water flux through aortic tissue”, Bull. Math. Biology 41 (1979) 79-90.

[22] M. Klanchar and J. M. Tarbell, "Modeling water flow through arterial tissue", Bull. Math. Biology 49 (1987) 651-669.

[23] W. M. Lai and V. C. Mow, "Drag induced compression of articular cartilage during a permeation experiment" Biorheology 17 (1980) 111-123.

[24] V. C. Mow and W. M. Lai, "Mechanics of animal joints", Ann. Rev. Fluid Mech. 11 (1979) 247-288.

[25] V. C. Mow, M. H. Holmes and W. M. Lai, "Fluid transport and mechanical properties of articular cartilage: a review", J. Biomechanics 17 (1984) 377-394.

[26] V. C. Mow, M. K. Kwan, W. M. Lai and M. H. Holmes, "A finite deformation theory for nonlinearly permeable soft hydrated biological tissues", in Frontiers in Biomechanics (eds. G. Schmid-Schoenbein, S. L-Y. Woo, B. W. Zweifach), (Springer-Verlag, 1985) 153-179.

[27] R. M. Nerem and J. F. Cornhill, "The role of fluid mechanics in artherogenesis", $J$. Biomech. Engng. 102 (1980) 181-189.

[28] G. Schettler, R. M. Nerem, H. Schmid-Schoenbein, H. Morl and C. Diehm (eds), Fluid Dynamics as a Localizing Factor in Atherosclerosis, (Springer-Verlag, Berlin 1983).

[29] K. Terzaghi, Erdbaumechanik auf Bodenphysikalischen Grundlagen, (Wien, Deuticke, 1925).

[30] S. Wolfram, Mathematica. A system for doing mathematics by computer, (AddisonWesley, U.S.A. 1998) 